\title{
Analysis of genes encoding epigenetic regulators in myeloproliferative neoplasms: Coexistence of a novel SETBP1 mutation in a patient with a $\mathrm{p} . \mathrm{V617F} J A K 2$ positive myelofibrosis
}

\author{
LAURA EDER-AZANZA ${ }^{1,2}$, CRISTINA HURTADO ${ }^{1,2}$, DAVID NAVARRO-HERRERA $^{1}$, \\ DIEGO CALAVIA $^{1}$, FRANCISCO JAVIER NOVO ${ }^{1,2}$ and JOSÉ LUIS VIZMANOS ${ }^{1,2}$ \\ ${ }^{1}$ Department of Biochemistry and Genetics, School of Sciences, University of Navarra; \\ ${ }^{2}$ Navarra Institute for Health Research (IdiSNA), E-31008 Pamplona, Spain
}

Received August 21, 2018; Accepted March 18, 2019

DOI: $10.3892 / \mathrm{mco} .2019 .1840$

\begin{abstract}
In recent years it has been shown that the causes of chronic myeloproliferative neoplasms (MPNs) are more complex than a simple signaling aberration and many other mutated genes affecting different cell processes have been described. For instance, mutations in genes encoding epigenetic regulators are more frequent than expected. One of the latest genes described as mutated is SET binding protein 1 (SETBPI). In silico tools have revealed that there are several human $S E T B P 1$ paralogous to nuclear receptor binding SET domain protein 1 (NSD1), NSD2 and NSD3, for example, which are also involved in the development of other hematological malignancies. Therefore, the present study analyzed the mutational status of NSD1, NSD2, NSD3 and SETBP1 in BCR-ABL1 negative MPNs with or without Janus kinase 2 (JAK2) p.V617F mutation. The present study revealed that the NSD genes are not frequently mutated in MPNs. However, a novel SETBPI mutation was identified in a patient with p.V617F JAK2 positive primary myelofibrosis. These results provide further insight into the genetic complexity of MPNs.
\end{abstract}

\section{Introduction}

Chronic myeloproliferative neoplasms (MPNs) have been considered diseases caused by aberrations in signaling, mainly affecting the JAK-STAT pathway. However, mutations in other genes that affect different cell processes have been described, suggesting that they are diseases with more complex causes. Recent findings suggest that MPNs are likely the result of

Correspondence to: Professor José Luis Vizmanos, Department of Biochemistry and Genetics, School of Sciences, University of Navarra, Research Building, C/Irunlarrea 1, E-31008 Pamplona, Spain E-mail: jlvizmanos@unav.es

Key words: primary myelofibrosis, epigenetic regulators, SET binding protein 1, Janus kinase 2, genetic complexity combined genetic dysregulation and can present mutations in epigenetic associated-genes such as TET2, ASXL1,EZH2, $I D H 1 / 2$ and DNMT3A (1-3) described also in the related myelodysplastic/myeloproliferative neoplasms (MDS/MPN).

SETBP1 encodes the SET binding protein 1, one component of the multi-functional SET complex in the nucleus that plays a role in histone modifications. In 2013, whole exome sequencing identified recurrent SETBPI mutations in approximately $25 \%$ patients with atypical chronic myeloid leukemia (aCML) and in a small proportion of patients with chronic myelomonocytic leukemia (CMML) and chronic neutrophilic leukemia (CNL) (4). SETBPl was the first gene recurrently mutated in aCML, a disease previously diagnosed by exclusion. All of the mutations described were located in a small region of 14 amino acids (858-871) in the highly-conserved SKI homologous region encoded by exon 4 of the gene.

SETBP1 seems to interact with SET protein, leading to the inhibition of the activity of PP2A and promoting the proliferation of leukemic cells (5). Overexpression of SETBPI increases the expression of other genes such as HOXA9 and HOXA10 leading to immortalization of murine myeloid progenitors (6), and mutant SETBP1 forms could drive the disease through similar mechanisms (7).

There are three SETBP1 paralogous genes (NSD1, NSD2 and NSD3) that encode proteins with similar functions, so that they could also be mutated in MPNs. Indeed, NSD1 and NSD3 are involved in fusions with NUP98 in myeloid malignancies. Nuclear receptor binding SET domain protein 1 (NSDI) encodes a H3K36 and H4K20 methyltransferase that activates or represses transcription depending on the cellular context. Some rare cases of acute myeloid leukemia (AML) with poor prognosis have at $(5 ; 11)(\mathrm{q} 35 ; \mathrm{p} 15)$ that fuses $N U P 98$ with $N S D 1$, and show abnormally high levels of H3K36 methylation and activation of transcription of oncogenes such as HOXA9 (8-10). On the other hand, nuclear receptor binding SET domain protein 3 (NSD3) encodes a H3K4 and H3K27 methyltransferase, marks of activation or repression of transcription, respectively. This gene has also been found fused with NUP98 in patients with AML (11) and therapy-related myelodysplastic syndrome (t-MDS) with poor prognosis (12). Finally, nuclear 
receptor binding SET domain protein 2 (NSD2) encodes an H3K36 histone methyltransferase that has also been implicated in gene fusions in multiple myeloma (13) and is mutated in pediatric acute lymphoblastic leukemia (ALL) (14). The p.E1099K mutation affects the SET domain and increases H3K36 dimethylation, which may lead to the transforming activity of NSD2 (14).

To date, little is known about the role of individual histone modifications in MPNs, and current research is focusing mainly on changes related to polycomb group proteins (3). These proteins can remodel chromatin; for example, polycomb repressive complex 2 (PRC2) catalyzes di- and trimethylation of H3K27 leading to gene silencing. Addition of sex combs like protein 1 (ASXLI) encodes an important mediator of PRC2 function, and mutations in this gene have been described in MDS/AML (15) and in MPNs (16). Thus, we asked whether SETBPI related genes could also be mutated in these diseases.

With this aim in mind, we analyzed the mutational profile of NSD1, NSD2 and NSD3 in a selected group of patients with MPNs.

\section{Materials and methods}

We performed denaturing high performance liquid chromatography (dHPLC) to analyze exon 13 of NSD1, NSD2 and $N S D 3$, which are homologous to SETBPI exon 4 in which mutations had been previously described. This SETBPI exon was also included in the analysis. In addition, we also analyzed exon 19 of NSD1 and NSD2 and exon 20 of NSD3, homologous to the NSD2-mutated exon in ALL (p.E1099K) (primers and conditions are shown in Table I).

We included samples from patients with different types of MPNs (PV, ET, PMF) and MDS/MPNs with and without the p.V617F mutation in Janus kinase 2 (JAK2). Ninety-two samples (34 ET, $37 \mathrm{PMF}$ and $21 \mathrm{MDS} / \mathrm{MPNs}$ ) were from patients without p.V617F JAK2 mutation, while 50 samples (8 ET, 8 PV, 4 PMF and 30 with an unspecified MPN type) harbored the p.V617F mutation. Finally, we also analyzed samples from 25 different cancer cell lines (Table II), most of them from hematological malignancies. As a control group, we included samples from 13 patients with B-ALL, the disease in which the p.E1099K NSD2 mutation had been described (14). Informed consent was obtained from all patients and procedures were approved by the Ethical Committee on Clinical Research of University of Navarra.

\section{Results}

Most of the nucleotide changes that we detected were SNPs already described (data not shown). No mis-sense changes were found in NSD1 and NSD3 so they do not seem to be frequently mutated in these diseases. With regard to NSD2, we did not detect any mis-sense mutations in samples from MPN and MDS/MPN patients, except for the previously described p.E1099K mutation that we observed in one patient with B-precursor ALL.

The analysis of SETBP1 found the mis-sense mutation p.S867G (c.2599A $>\mathrm{G}$ according the first nucleotide of the translation start codon of the CDS from NM_015559) (Fig. 1) in the sample from one patient with a p.V617F JAK2 PMF This change is located in a residue that is highly conserved between different species in the SKI homologous region, the mutational hotspot described previously In silico analysis by several bioinformatics tools like PolyPhen-2, MutPred2, SIFT and FATHMM showed that it is probably damaging (data not shown). No additional material was available to determine the somatic status of this change. This mutation has not been described as a polymorphism or a cancer-related mutation in other databases. In fact, in COSMIC (Catalogue of Somatic Mutations in Cancer, cancer.sanger.ac.uk/cosmic) there are other variants affecting the same codon but not this specific nucleotide. These mutations are p.S867N (c.2600G $>\mathrm{A}$ ) a somatic change predicted as pathogenic and found in an intestinal adenocarcinoma, p.S867R (c.2601C $>$ A) a non-confirmed somatic change predicted as neutral and found in four samples from MDS and AML associated with MDS and the silent p.S867S (c.2601C >T), a somatic change predicted also as neutral and found in two samples from stomach and intestine with adenocarcinoma but described as a SNP in dbSNP (rs376371660). This is also the only change in ExAC database (Exome Aggregation Consortium, http://exac.broadinstitute.org) affecting this codon, found in 7 of 120746 alleles with a frequency of 0.00005797 . This is the only variant also described in 1000 Genomes Phase 3 database ( $w w w . i n t e r n a t i o n a l g e n o m e . o r g / c a t e g o r y / p h a s e-3$ ) with a frequency of 0.00039936 and in the ESP database (Exome Sequencing Project, evs.gs.washington.edu) with a frequency of 0.00007688 , in both cases with only one allele $\mathrm{T}$ found. In gnomAD (Genome Aggregation Database, gnomad. broadinstitute.org), that spans 125,748 exome sequences and 15,708 whole-genome sequences from unrelated individuals the mutation p.S867G is also not described.

\section{Discussion}

SETBP1 mutations can coexist with aberrations in other genes frequently mutated in myeloid neoplasms such as $C B L, A S X L 1$, $U 2 A F 1$ or $R U N X 1 \quad(3,17,18)$ thus cooperating in the progression of the disease. However, the coexistence of mutations in SETBPI and JAK2 in MPNs has not been described to date. In the case of $A S X L 1$ and $S E T B P 1$ mutations, the former may initially inhibit cell differentiation while mutations in SETBPI would provide a proliferative advantage (18). Although a direct physical interaction between CBL and SETBP1 has not been demonstrated, it seems plausible that both mutations could cooperate indirectly by reducing cytokine dependence of leukemia cells (17).

Furthermore, it is well established that mutations in genes related to epigenetic regulation of gene expression can coexist with mutations in $J A K 2$ and could modulate the progression of the disease $(3,19)$. For instance, TET2 encodes the Tet methylcytosine dioxygenase 2 which catalyzes the conversion of the 5-methylcytosine $(5 \mathrm{mC})$ into 5-hydroxymethylcytosine $(5 \mathrm{hmC})$ and plays a key role in active DNA demethylation and it is frequently mutated in patients with MPNs (20). TET2 mutations have been found in $17 \%$ of patients with p.V617F $J A K 2$ (20). Although both mutations are present in different clones, data published to date show that TET2 somatic mutations might be associated with increased aggressiveness and 
Table I. Polymerase chain reaction primers used for the mutational analysis of NSD1, NSD2, NSD3 and SETBP1 by dHPLC.

\begin{tabular}{|c|c|c|c|c|c|}
\hline Primer & $\operatorname{Tm}\left({ }^{\circ} \mathrm{C}\right)$ & $\% \mathrm{GC}$ & Sequence $\left(5^{\prime} \rightarrow 3^{\prime}\right)$ & PCR product (bp) & dHPLC conditions $\left({ }^{\circ} \mathrm{C}\right)$ \\
\hline NSD1-e13L & 61.0 & 29.17 & AATTTATCTTCTTTTGGCTTCTCA & 285 & $57.3 ; 59.2 ; 61$ \\
\hline NSD1-e13R & 62.8 & 40.91 & TCTGTTGCCAATTAAACTGAGG & & \\
\hline NSD1-e13Lm & 63.7 & 33.33 & 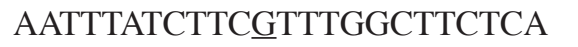 & & \\
\hline NSD1_e19L & 66.2 & 54.55 & TGCTGCTGACAGTGGTAGGAGT & 350 & $53.1 ; 56.8 ; 55.8$ \\
\hline NSD1_e19R & 65.8 & 39.13 & CAGTGAAAACAGCATTTCCCATT & & \\
\hline NSD1_e19Lm & 70.3 & 59.10 & TGCTGCTGACGGGTGGTAGGAGT & & \\
\hline NSD2_e13L & 65.4 & 54.55 & АССТСТСТСТССАССССТТСТТ & 329 & $57.8 ; 58.7 ; 62.7$ \\
\hline NSD2_e13R & 70,1 & 54.55 & ACAATCCCAACAGCCCACCTTC & & \\
\hline NSD2_e13Rm & 73.7 & 59.10 & ACAATCCCAACGGCCCACCTTC & & \\
\hline NSD2_e19L & 69.6 & 54.55 & TCATGATGGGGAGTCTTGAGCC & 285 & $61.2 ; 61.8$ \\
\hline NSD2_e19R & 68.5 & 54.55 & CCACAGGGCAAAGTCCAGTTCT & & \\
\hline NSD2_e19Rm & 64.3 & 50.00 & CCACAGGTCAAAGTCCAGTTCT & & \\
\hline NSD3_e13L & 64.6 & 47.83 & GCTGTTTGATGTCTGTAGCTGCT & 308 & $57.3 ; 59.4 ; 60.6$ \\
\hline NSD3_e13R & 66,7 & 41.67 & TCTTTGTCTCCTTCTTCAGCTGTT & & \\
\hline NSD3_e13Lm & 64,3 & 52.17 & GCTGTTTGGTGTCTGTAGCTGCT & & \\
\hline NSD3_e20L & 61.9 & 37.50 & ACCATTTCTTTCTAGGGTTGAAGT & 372 & $53.8 ; 56 ; 60$ \\
\hline NSD3_e20R & 62.8 & 41.67 & TCTTTGTCTCCTTCTTCAGCTGTT & & \\
\hline NSD3_e20Lm & 64,3 & 41.67 & ACCATTTCT $\underline{G T C T A G G G T T G A A G T ~}$ & & \\
\hline SETBP1-e4F9L & 65.8 & 40.91 & СТСТТССААССААААСССАААА & 394 & $60.7 ; 62$ \\
\hline SETBP1-e4F9R & 65.4 & 45.45 & CTTTTCCGTTTCCTCTTGTGCT & & \\
\hline SETBP1-e4F9Rm & 61.2 & 40.91 & CTTTTCCGTTT杰TCTTGTGCT & & \\
\hline
\end{tabular}

Forward and reverse primers used to amplify the DNA sequences of interest. The mutations designed to create a control mutant product are underlined. The length of the PCR product is shown in the fifth column. Finally, melting temperature conditions (dHPLC) for each exon are also shown. NSD, nuclear receptor binding SET domain protein 1; SETBP1, SET binding protein 1; dHPLC, denaturing high performance liquid chromatography; PCR, polymerase chain reaction.

higher frequency of organomegaly in ET patients, but not in PV or PMF patients (19). Although SETBPl mutations have not been associated with mutations in JAK2 in MPNs to date, our finding suggests the possibility of a similar interplay of mutational mechanisms.

Recently two cases have been reported with SchinzelGiedion syndrome (SGS, MIM 269150) and a milder phenotype with the mutation at this residue p.S867R $(21,22)$. SGS is a rare dominant developmental disorder characterized by multiple malformations including midface hypoplasia, cardiac defects, hydronephrosis and skeletal abnormalities. It seems caused by germline de novo mutations in SETBP1 in a hotspot of $12 \mathrm{bp}$ coding for residues 868 to 871 of the protein, that overlaps to the somatic mutations reported in myeloid malignancies and support a gain-of-function effect. This dual role in cancer and development is not new, there are other genes such as $H R A S$, ASXL1, EZH2 and FGFR2 in which germline mutations cause developmental disorders and the overlapping somatic mutations drive cancer. The recurrently mutated region (868-871) in SETBP1 is highly conserved and it has been identified as important to initiate degradation by ubiquitination so the mutations could increase protein stability $(4,21,22)$. The milder phenotype shown by both patients with SGS and the p.S867R would point to a less activating gain-of-function of this mutant protein.
The sample available in our case did not allow us to determine whether SETBP1 and JAK2 mutations coexist in the same clone. Lack of access to clinical data of this patient prevents us from drawing definitive conclusions about the putative effects of their presence in the progression of the disease. SETBPI mutations usually have poor prognosis and are frequently acquired as secondary events in other myeloid neoplasms (PMF can arise on its own or as a progression of PV or ET) (18), so the p.S867G mutation in SETBP1 could be relevant for the progression of the disease. In addition, additional experiments would be required to demonstrate the pathogenicity of the p.S867G mutation. These experiments should be performed on cell lines that stably express p.S867G SETBPI and this mutation in combination with the p.V617F JAK2 to test their possible cooperation for the transformation of the disease.

In this study we show that NSD1, NSD2 and NSD3 genes, that are paralogous to $S E T B P 1$ and encode also epigenetic regulators, are not frequently mutated in MPNs in the SETBP1 homologous region. However, we show that the p.V617F JAK2 can coexist with mutations in SETBP1, a gene mutated in some myeloid neoplasms. This fact adds support to the notion that these diseases are genetically complex and shows the need to identify additional oncogenic mechanisms that could promote progression or phenotypic diversity, in order to design new targeted therapies. 
Table II. Human cancer cell lines tested.

\begin{tabular}{|c|c|c|}
\hline Cell line name & Repository (number) & Origin \\
\hline A-549 & DSMZ (ACC-107) & Lung carcinoma \\
\hline BK-006 & ECACC & p.V617F JAK2 positive PV \\
\hline BK-013 & ECACC (98100924) & p.V617F JAK2 negative ET \\
\hline BK015 & ECACC (99092421) & p.V617F JAK2 negative ET \\
\hline DAUDI & DSMZ (ACC-78) & Burkitt lymphoma \\
\hline EOL-1 & DSMZ (ACC-386) & Acute myeloid eosinophilic leukemia \\
\hline F-36P & DSMZ (ACC-543) & Acute myeloid leukemia secondary to myelodysplastic syndrome (MDS) \\
\hline HCC-1937 & DSMZ (ACC-513) & Breast carcinoma \\
\hline HEL & DSMZ ACC-11 & Erythroleukemia \\
\hline HL60 & DSMZ ACC-3 & Acute myeloid leukemia (AML) \\
\hline HU3 & Dr Morgan, USA & Acute megakaryoblastic leukemia \\
\hline K-562 & DSMZ ACC-10 & $B C R-A B L 1$ positive chronic myeloid leukemia (CML) in blast crisis \\
\hline KARPAS-299 & DSMZ ACC-31 & T cell lymphoma \\
\hline KARPAS-422 & DSMZ ACC-32 & B cell lymphoma \\
\hline M-07e & DSMZ ACC-104 & Acute megakaryoblastic leukemia \\
\hline MG-63 & ATCC: CRL-1427 & Osteosarcoma \\
\hline MOLM-13 & DSMZ ACC-554 & Acute myeloid leukemia (AML) \\
\hline MOLT-16 & DSMZ ACC-29 & T cell acute lymphoid leukemia (T-ALL) \\
\hline MOLT-4 & DSMZ ACC-362 & Acute lymphoid leukemia (ALL) \\
\hline MV-411 & DSMZ ACC-102 & Acute monoblastic/monocytic leukemia \\
\hline RAJI & DSMZ ACC-319 & Burkitt lymphoma \\
\hline REH & DSMZ ACC-22 & B-ALL (t(12;21) (p13;q22) (fusion ETV6-RUNX1)) \\
\hline SET2 & DSMZ ACC-608 & p.V617F JAK2 positive ET \\
\hline TF-1 & DSMZ ACC-334 & Erythroleukemia \\
\hline UKE-1 & $\begin{array}{l}\text { Dr W. Fiedelr, Hamburg, } \\
\text { Germany }\end{array}$ & p.V617F JAK2 positive ET transformed to AML \\
\hline
\end{tabular}

ECACC, European Collection of Cell Culture; DSMZ, Deutsche Sammlung von Mikroorganismen und Zellkulturen.

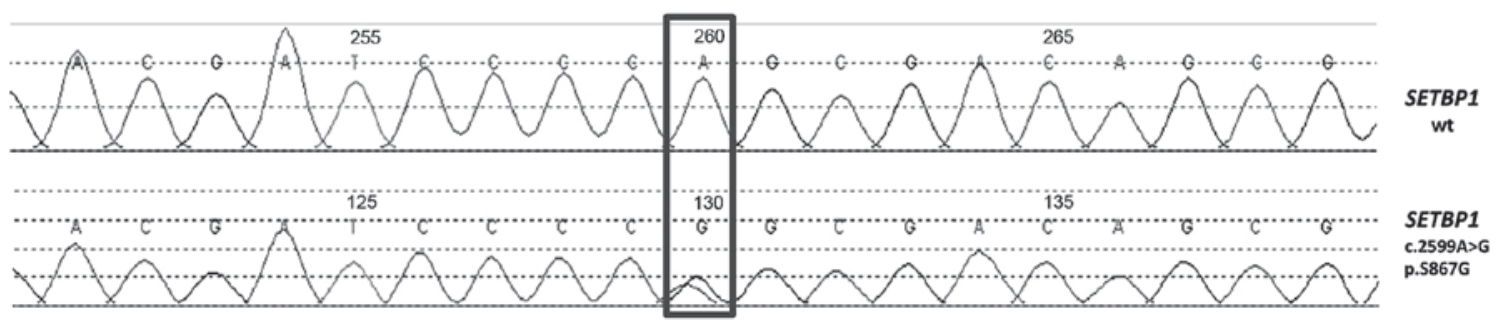

Figure 1. Electropherograms of the sequence surrounding the mutation c.2599A $>\mathrm{G}$ (p.S867G). Up, wild-type sequence; down, sequence from the patient. SETBP1, SET binding protein 1.

\section{Acknowledgements}

Not applicable.

\section{Funding}

The present study was funded by the PIUNA Program of the University of Navarra. LE and DN have PhD Studentships granted by the Ministry of Education, Culture and Sports of Spain (grant nos. FPU14/03669 and FPU13/00424 respectively) within the University Faculty Training (FPU) program.

\section{Availability of data and materials}

The datasets used and/or analyzed during this study are available from the corresponding author on reasonable request.

\section{Authors' contributions}

LEA, FJN and JLV designed the study. LEA and CH performed the experiments. LEA, DNH, DC and JLV analyzed the data. LEA, FJN and JLV wrote the manuscript. All authors have read and approved the final manuscript. 


\section{Ethics approval and consent to participate}

Written informed consent was obtained from all of the patients and the procedures were approved by the Ethical Committee on Clinical Research of University of Navarra (Pamplona, Spain).

\section{Patient consent for publication}

Not applicable.

\section{Competing interests}

The authors declare that they have no competing interests.

\section{References}

1. Murati A, Brecqueville M, Devillier R, Mozziconacci MJ, Gelsi-Boyer V and Birnbaum D: Myeloid malignancies: Mutations, models and management. BMC Cancer 12: 304, 2012

2. Shih AH, Abdel-Wahab O, Patel JP and Levine RL: The role of mutations in epigenetic regulators in myeloid malignancies. Nat Rev Cancer 12: 599-612, 2012.

3. McPherson S, McMullin MF and Mills K: Epigenetics in myeloproliferative neoplasms. J Cell Mol Med 21: 1660-1667, 2017.

4. PiazzaR, VallettaS, WinkelmannN, RedaelliS,Spinelli R,Pirola A, Antolini L, Mologni L, Donadoni C, Papaemmanuil E, et al: Recurrent SETBP1 mutations in atypical chronic myeloid leukemia. Nat Genet 45: 18-24, 2013.

5. Cristóbal I, Blanco FJ, Garcia-Orti L, Marcotegui N, Vicente C, Rifon J, Novo FJ, Bandres E, Calasanz MJ, Bernabeu C and Odero MD: SETBP1 overexpression is a novel leukemogenic mechanism that predicts adverse outcome in elderly patients with acute myeloid leukemia. Blood 115: 615-625, 2010.

6. Oakley K, Han Y, Vishwakarma BA, Chu S, Bhatia R, Gudmundsson KO, Keller J, Chen X, Vasko V, Jenkins NA, et al: Setbp1 promotes the self-renewal of murine myeloid progenitors via activation of Hoxa9 and Hoxa10. Blood 119: 6099-6108, 2012.

7. Trimarchi T, Ntziachristos P and Aifantis I: A new player SETs in myeloid malignancy. Nat Genet 45: 846-847, 2013.

8. Akiki S, Dyer SA, Grimwade D, Ivey A, Abou-Zeid N, Borrow J, Jeffries S, Caddick J, Newell H, Begum S, et al: NUP98-NSD1 fusion in association with FLT3-ITD mutation identifies a prognostically relevant subgroup of pediatric acute myeloid leukemia patients suitable for monitoring by real time quantitative PCR. Genes Chromosomes Cancer 52: 1053-1064, 2013.

9. Hollink IH, van den Heuvel-Eibrink MM, Arentsen-Peters ST, Pratcorona M, Abbas S, Kuipers JE, van Galen JF, Beverloo HB, Sonneveld E, Kaspers GJ, et al: NUP98/NSD1 characterizes a novel poor prognostic group in acute myeloid leukemia with a distinct HOX gene expression pattern. Blood 118: 3645-3656, 2011.
10. Shiba N, Ichikawa H, Taki T, Park MJ, Jo A, Mitani S, Kobayashi T, Shimada A, Sotomatsu M, Arakawa H, et al: NUP98-NSD1 gene fusion and its related gene expression signature are strongly associated with a poor prognosis in pediatric acute myeloid leukemia. Genes Chromosomes Cancer 52: 683-693, 2013.

11. Rosati R, La Starza R, Veronese A, Aventin A, Schwienbacher C, Vallespi T, Negrini M, Martelli MF and Mecucci C: NUP98 is fused to the NSD3 gene in acute myeloid leukemia associated with $\mathrm{t}(8 ; 11)(\mathrm{p} 11.2 ; \mathrm{p} 15)$. Blood 99: 3857-3860, 2002.

12. Taketani T, Taki T, Nakamura H, Taniwaki M, Masuda J and Hayashi Y: NUP98-NSD3 fusion gene in radiation-associated myelodysplastic syndrome with $\mathrm{t}(8 ; 11)(\mathrm{p} 11 ; \mathrm{p} 15)$ and expression pattern of NSD family genes. Cancer Genet Cytogenet 190: 108-112, 2009.

13. Chesi M, Nardini E, Lim RS, Smith KD, Kuehl WM and Bergsagel PL: The $\mathrm{t}(4 ; 14)$ translocation in myeloma dysregulates both FGFR3 and a novel gene, MMSET, resulting in IgH/MMSET hybrid transcripts. Blood 92: 3025-3034, 1998

14. Jaffe JD, Wang Y, Chan HM, Zhang J, Huether R, Kryukov GV, Bhang HE, Taylor JE, Hu M, Englund NP, et al: Global chromatin profiling reveals NSD2 mutations in pediatric acute lymphoblastic leukemia. Nat Genet 45: 1386-1391, 2013.

15. Gelsi-Boyer V, Trouplin V, Adélaïde J, Bonansea J, Cervera N, Carbuccia N, Lagarde A, Prebet T, Nezri M, Sainty D, et al: Mutations of polycomb-associated gene ASXL1 in myelodysplastic syndromes and chronic myelomonocytic leukaemia. $\mathrm{Br}$ J Haematol 6: 788-800, 2009.

16. Abdel-Wahab O, Tefferi A and Levine RL: Role of TET2 and ASXL1 mutations in the pathogenesis of myeloproliferative neoplasms. Hematol Oncol Clin North Am 5: 1053-1064, 2012.

17. Makishima H, Yoshida K, Nguyen N, Przychodzen B, Sanada M, Okuno Y, Ng KP, Gudmundsson KO, Vishwakarma BA, Jerez A, et al: Somatic SETBP1 mutations in myeloid malignancies. Nat Genet 45: 942-946, 2013.

18. Makishima H: Somatic SETBP1 mutations in myeloid neoplasms. Int J Hematol 105: 732-742, 2017.

19. Saeidi K: Myeloproliferative neoplasms: Current molecular biology and genetics. Crit Rev Oncol Hematol 98: 375-389, 2016.

20. Tefferi A, Pardanani A, Lim KH, Abdel-Wahab O, Lasho TL, Patel J, Gangat N, Finke CM, Schwager S, Mullally A, et al: TET2 mutations and their clinical correlates in polycythemia vera, essential thrombocythemia and myelofibrosis. Leukemia 23: 905-911, 2009.

21. Carvalho E, Honjo R, Magalhães M, Yamamoto G, Rocha K, Naslavsky M, Zatz M, Passos-Bueno MR, Kim C and Bertola D: Schinzel-Giedion syndrome in two Brazilian patients: Report of a novel mutation in SETBP1 and literature review of the clinical features. Am J Med Genet 167A: 1039-1046, 2015.

22. Acuna-Hidalgo R, Deriziotis P, Steehouwer M, Gilissen C, Graham SA, van Dam S, Hoover-Fong J, Telegrafi AB, Destree A, Smigiel R, et al: Overlapping SETBP1 gain-of-function mutations in Schinzel-Giedion syndrome and hematologic malignancies. PLoS Genet 13: e1006683, 2017. 